\title{
Utilização de serviços de atenção básica à saúde por caminhoneiros
}

\author{
Helisamara Mota Guedes, M.Sc.*, Alessandra Custódio de Paula**, Aline Mara da Conceição Silva**, \\ Martha Elisa Ferreira de Almeida, M.Sc.***
}

${ }^{*}$ Enfermeira, Professora Assistente II da Universidade Federal dos Vales do Jequitinhonha e Mucuri - UFVJ-MG, **Enfermeira pelo Centro Universitário do Leste de Minas Gerais - Unileste-MG, ${ }^{* * *}$ Nutricionista, Professora Assistente II da Universidade Federal de Viçosa, Campus de Rio Paranaíba-CRP/UFV

\section{Resumo}

O objetivo foi verificar a adesão de caminhoneiros aos serviços de Atenção Básica à Saúde. Trata-se de um estudo quantitativo, cujo instrumento de coleta de dados foi um formulário semiestruturado. Os 248 participantes foram questionados sobre o seu conhecimento acerca dos serviços oferecidos pela Atenção Básica à Saúde, dos quais $151(60,8 \%)$ disseram conhecêlos. Os serviços mais citados e utilizados foram: consulta médica, vacinação e controle da pressão arterial. 65 (26,2\%) dos caminhoneiros afirmaram que não utilizavam nenhum dos serviços. Quanto à forma de tratamento de problemas de saúde, apenas $73(29,4 \%)$ dos avaliados procuravam o Posto de Saúde, sendo a presença de Postos de Saúde nas estradas a sugestão mais citada para melhorar o atendimento à saúde. Entre outras atribuiçóes, cabe aos enfermeiros criar estratégias para atrair esta população aos serviços de saúde, uma vez que a promoção da saúde trará benefício para esta categoria de trabalhadores e, por extensão, para toda a sociedade.

Palavras-chave: Serviços Básicos de Saúde, cuidados de enfermagem, Sistema Único de Saúde.

\section{Abstract \\ Truck drivers access to Primary Health Care Services}

The objective of this study was to verify the adhesion of truck drivers to Primary Health Care Services. This was a quantitative study, using a semistructured questionnaire to collect data. 248 participants were asked about their knowledge about services offered at Primary Health Care, and $151(60.8 \%)$ said they know them. The most cited and used services were: medical consultation, vaccination and blood pressure control. 65 (26.2\%) truck drivers said they did not use these services. In relation to the way to treat health problems, only 73 (29.4\%) attended Health Center, and suggested that Health Units should be more accessible on the roads in order to improve health care. Among other duties, nurses should create strategies to attract this population and convince them to use health services, since health promotion will benefit these workers and for extension the society as a whole.

Key-words: Basic Health Services, nursing care, Unified Health System. 


\section{Resumen}

\section{Utilización de servicios de salud en atención primaria por camioneros}

El objetivo fue verificar la adhesión de los camioneros a los servicios de salud en atención primaria. Se trata de un estudio cuantitativo que utilizó un formulario semiestructurado para recolectar los datos. A los 248 participantes se les preguntó sobre sus conocimientos acerca de los servicios que ofrecen los servicios de salud en atención primaria, y 151 (60.8\%) dijeron que los conocen. Los servicios más citados y más utilizados fueron: consulta médica, vacunación y control de la presión arterial. 65 (26.2\%) de los camioneros dijeron que no utilizan ningún servicio. Con relación a la forma de tratar los problemas de salud, sólo 73 (29.4\%) de los participantes buscaron el Centro de Salud, y sugirieron que debería haber mayor accesibilidad a las unidades de salud en las carreteras para mejorar la atención a la salud. Entre otras atribuciones, corresponde a los enfermeros crear estrategias para atraer esta población a los servicios de salud, ya que la promoción de la salud beneficiará estos trabajadores y por extensión toda la sociedad.

Palabras-clave: Servicios Básicos de Salud, atención de enfermería, Sistema Único de Salud.

\section{Introdução}

O Brasil possui uma frota aproximada de 1,8 milhôes de caminhôes e uma população de mais de 700.000 motoristas caminhoneiros [1]. Estima-se que $58 \%$ do transporte de cargas brasileiras seja realizado pelas rodovias, o que evidencia a importância desta profissão [2].

Pressionados por fatores socioeconômicos, como dívidas pessoais e exigências de entrega de cargas em curto prazo, muitos caminhoneiros chegam a rodar mais de 18 horas por dia [3], sendo o grande período que passam fora de casa um facilitador para práticas de risco, como o grande número de parceiras (os) sexuais e o uso de drogas como o "rebite" [4-5].

Os caminhoneiros podem estar mais expostos a riscos cardiovasculares devido às características peculiares de sua profissão, considerando que esses profissionais se alimentam, de modo geral, em restaurantes à beira das estradas, onde há uma grande oferta de alimentos com alto teor calórico e baixo teor nutritivo, além de bebidas alcoólicas [5-6].

A Atenção Básica à Saúde é caracterizada como um conjunto de açóes de saúde destinadas ao âmbito individual e coletivo da população. Abrange a promoção da saúde e a proteção de agravos, o diagnóstico, o tratamento, a reabilitação e a manutenção da saúde, considerando o sujeito em sua singularidade e também na sua complexidade, integralidade e inserção sociocultural, tratando problemas que possam comprometer suas possibilidades de viver de forma saudável [7].

Neste contexto, este estudo teve como objetivo verificar a adesáo dos caminhoneiros que trafegavam na BR-381, em Minas Gerais, aos serviços da Atenção Básica a Saúde.

\section{Material e métodos}

Para a realização da pesquisa, de caráter transversal com abordagem quantitativa, foi aplicado um estudo piloto com 10 condutores de veículos, o que subsidiou o cálculo do tamanho da amostra pelo programa Diman 1.0. Foi definida uma amostra de 128 caminhoneiros, porém, para dar maior confiabilidade aos dados foram entrevistados 248 caminhoneiros que trafegavam na BR-381, abordados no posto de combustível Torque Diesel Ltda., localizado no município de Timóteo/MG.

Os critérios de inclusão dos pesquisados foram: ter um ano ou mais de exercício da profissão e concordar em participar do estudo através da assinatura do Termo de Consentimento Livre e Esclarecido, com base na Resoluçáo 196/96 [8]. A coleta de dados ocorreu do dia 1 ao dia 12 de junho de 2008, no horário das 13:00 às 17:00 horas. A escolha do posto de combustível foi realizada com base na presença dos seguintes setores: serviço de abastecimento, borracharia, loja de peças, troca de óleo, restaurante, lanchonete, banheiros com duchas dentro do próprio posto, presença de uma transportadora de cargas onde se concentravam muitos caminhoneiros à espera de cargas e um amplo estacionamento para o descanso desses profissionais.

O caminhoneiro era abordado, individualmente, à media que chegava ao posto. Após a assinatura do TCLE, os participantes foram encaminhados a um local reservado, anexo ao escritório geral do posto, onde todos os procedimentos foram explicados. O instrumento de coleta de dados utilizado foi um formulário semiestruturado, contendo 10 questôes, cuja aplicação, de forma individualizada, durou, em média, 15 minutos. Nos itens relativos 
ao conhecimento e utilização dos serviços oferecidos pelas Unidades Básicas de Saúde, formas de acesso a esses serviços e sugestóes para melhorar o atendimento de saúde, os participantes marcaram mais de uma resposta.

Os dados foram tabulados, expressos em frequência absoluta e relativa e apresentados em tabelas e figuras.

O estudo foi aprovado pelo Comitê de Ética em Pesquisa do Centro Universitário do Leste de Minas Gerais - Unileste/MG, com o parecer $\mathrm{n}^{\circ}$ 13.57.08.

\section{Resultados e discussão}

Participaram do estudo 248 caminhoneiros, sendo $99,6 \%$ do sexo masculino e $0,4 \%$ do sexo feminino. A faixa etária que predominou no estudo foi de 30 a 39 anos (Tabela I), e os idosos representavam 3,8\% dos pesquisados. Em relação ao estado civil, observou-se que 73,0\% eram casados. Quando perguntados em quais regióes brasileiras residiam, $92,8 \%$ relataram ser na região Sudeste, seguida pelas regiốes Sul (5,2\%) e Nordeste (2,0\%). A maioria dos caminhoneiros (47,1\%), com relaçáo à escolaridade, não concluiu o Ensino Fundamental, 59 (23,8\%) cursaram todo esse nível de ensino e 48 (19,3\%) terminaram o Ensino Médio.

Enquanto neste estudo a faixa etária predominante foi de 30 a 39 anos, em estudo realizado com motoristas de caminhão no interior de São Paulo [9], a maioria tinha entre 40 e 49 anos; apenas 3,8\% dos pesquisados eram idosos, sendo a idade neste grupo um importante fator de risco para as doenças crônicas não transmissíveis [1]. Tal porcentagem de pesquisados idosos foi semelhante àqueles observados em estudo com caminhoneiros da cidade de Santos/SP [4] e do interior de Sáo Paulo [9], onde 2,9 e 5,0\%, respectivamente, eram idosos.

A maioria dos entrevistados eram casados. Este dado também foi observado no estudo feito por Villarinho et al. [4], no qual 81,4\% dos motoristas eram casados e em outra pesquisa realizada no Rio de Janeiro para analisar a qualidade de vida de 206 caminhoneiros, quando identificou-se que 76,6\% eram casados [10]. Sugere-se que, pelo fato de ser casado, o cônjuge incentiva mais o motorista sobre a prevenção e/ou tratamento das doenças, gerando como consequência uma maior busca aos serviços de Atenção Básica à Saúde.
O Ensino Fundamental não foi concluído pela maioria, entretanto, nos estudos de Villarinho et al. [4] e Nascimento [11] foi observado que a maioria dos motoristas avaliados possuíam o Primeiro Grau completo, o que levou tais autores a sugerir que o nível de escolaridade pode impactar diretamente sobre os hábitos de saúde do indivíduo. Entre os motoristas profissionais, o risco de doenças cardiovasculares e musculoesqueléticas varia em função da idade e dos anos de escolaridade dos motoristas, com risco aumentado para os trabalhadores dos setores de cargas e de passageiros [9].

Tabela I - Frequência absoluta e relativa de motoristas que trafegam na BR-381 quanto ao perfil sociodemográfico. Timóteo/MG, 2008.

\begin{tabular}{|c|c|c|}
\hline Variável & $\mathrm{n}$ & $\%$ \\
\hline \multicolumn{3}{|l|}{ Idade (anos) } \\
\hline $21-29$ & 37 & 14,8 \\
\hline $30-39$ & 88 & 35,4 \\
\hline $40-49$ & 75 & 30,4 \\
\hline $50-59$ & 39 & 15,6 \\
\hline$\geq 60$ & 9 & 3,8 \\
\hline \multicolumn{3}{|l|}{ Estado civil } \\
\hline Casado & 181 & 73,0 \\
\hline Solteiro & 51 & 20,6 \\
\hline Separado & 15 & 6,0 \\
\hline Viúvo & 1 & 0,4 \\
\hline \multicolumn{3}{|l|}{ Escolaridade } \\
\hline Ensino Fundamental incompleto & 117 & 47,1 \\
\hline Ensino Fundamental completo & 59 & 23,8 \\
\hline Ensino Médio incompleto & 18 & 7,2 \\
\hline Ensino Médio completo & 48 & 19,3 \\
\hline Ensino Superior incompleto & 2 & 1,0 \\
\hline Ensino Superior completo & 4 & 1,6 \\
\hline \multicolumn{3}{|l|}{ Média de tempo fora de casa (dias) } \\
\hline Durante o dia & 17 & 6,8 \\
\hline $1-5$ & 79 & 32,0 \\
\hline $6-10$ & 91 & 36,5 \\
\hline $11-15$ & 30 & 12,0 \\
\hline $16-20$ & 10 & 4,0 \\
\hline $21-25$ & 2 & 0,8 \\
\hline $26-30$ & 12 & 5,0 \\
\hline$\geq 31$ & 7 & 2,9 \\
\hline \multicolumn{3}{|l|}{ Tempo de profissão (anos) } \\
\hline $1-5$ & 45 & 18,0 \\
\hline $6-10$ & 39 & 15,7 \\
\hline $11-15$ & 41 & 16,5 \\
\hline $16-20$ & 43 & 17,3 \\
\hline $21-25$ & 31 & 12,5 \\
\hline $26-30$ & 22 & 8,8 \\
\hline$\geq 31$ & 27 & 11,2 \\
\hline
\end{tabular}


Quando questionados sobre a média de tempo em que ficavam fora de casa por conta do serviço, a maioria relatou ficar de 6 a 10 dias (36,5\%), seguidos dos que ficavam de 1 a 5 dias; já 2,9\% dos entrevistados afirmaram ficar mais de 31 dias longe de casa, esperando cargas para serem transportadas. Segundo relatos de alguns caminhoneiros durante a entrevista, "longos períodos longe de casa culminam com o sentimento de insatisfação por não acompanharem de perto o crescimento e o desenvolvimento dos filhos, e a rotina da família”. O período de tempo de exercício da profissão que teve a maior percentagem de caminhoneiros foi o de 1 a 5 anos, em seguida aparecendo o período de 16 a 20 anos. O caminhoneiro com maior experiência no trabalho registrou 50 anos de estrada. Observou-se, através de relatos, que a escolha da profissão se deu por motivação pessoal e por influência familiar, e que o tempo de profissão estava ligado, principalmente, à necessidade de prover recursos básicos para o sustento da família.

Com relação à ausência do domicílio por causa do trabalho, a maioria relatou ser de 6 a 10 dias, enquanto no estudo de Villarinho et al. [4] 62,0\% dos avaliados ficavam somente até 24 horas fora de casa. Quanto ao período de tempo de profissáo, a maior percentagem foi de caminhoneiros com 1 a 5 anos, enquanto no estudo de Nascimento et al. [3] a maioria desses profissionais $(81,0 \%)$ tinha acima de 10 anos de profissáo.

Quando questionados sobre o conhecimento dos serviços oferecidos pela Atenção Básica à Saúde do Sistema Único de Saúde (SUS), 151 (60,8\%) disseram conhecer os serviços, enquanto 97 (39,2\%) disseram náo conhecer. Os serviços mais conhecidos eram o de consulta médica $(28,7 \%)$, vacinação $(27,2 \%)$ e controle da pressão arterial $(14,2 \%)$ (Tabela II). A pesquisa feita por Guedes et al. [5] registrou que somente $53,85 \%$ dos caminhoneiros buscavam a Unidade Básica de Saúde.

A não-utilização de qualquer um dos serviços oferecidos pelo SUS foi relatada por $65(26,2 \%)$ dos caminhoneiros, por não apresentar qualquer necessidade de saúde que os fizesse buscar os mesmos. Ao serem interrogados se buscavam os serviços de saúde do SUS, 133 (53,5\%) afirmaram não buscar o serviço nos últimos 12 meses, enquanto 115 $(46,5 \%)$ disseram que buscavam esses serviços, e destes, $78(31,4 \%)$ frequentavam somente quando estavam doentes, $20(8,1 \%)$ anualmente, $11(4,4 \%)$ mensalmente e 6 semestralmente. Os serviços mais utilizados foram o de consulta médica $(33,1 \%)$, o de vacinação $(34,9 \%)$ e o de controle da pressão arterial $(15,7 \%)$ (Tabela II).

Tabela II - Frequência absoluta e relativa de caminhoneiros segundo o seu conhecimento e utilização dos serviços oferecidos pelas Unidades Básicas de Saúde. Timóteo/MG, 2008.

\begin{tabular}{lcccc}
\hline \multicolumn{1}{c}{ Serviços } & $\begin{array}{c}\text { Conhecimen- } \\
\text { to sobre os } \\
\text { serviços }\end{array}$ & $\begin{array}{c}\text { Utilização dos } \\
\text { serviços }\end{array}$ \\
\cline { 2 - 5 } & $\mathrm{N}$ & $\%$ & $\mathrm{n}$ & $\%$ \\
\hline Consulta médica & 119 & 28,7 & 55 & 33,1 \\
Vacinação & 113 & 27,2 & 58 & 34,9 \\
Controle da pressão & 59 & 14,2 & 26 & 15,7 \\
arterial & 47 & 11,3 & 11 & 6,6 \\
Curativo & 36 & 8,7 & 9 & 5,5 \\
Saúde bucal & 20 & 4,8 & 2 & 1,2 \\
Controle do diabetes & & & & \\
mellitus & 11 & 2,7 & 1 & 0,6 \\
Consulta de enferma- & & & & \\
gem & 7 & 1,7 & - & - \\
Saúde do idoso & 3 & 0,7 & 4 & 2,4 \\
Exames & & & & \\
\hline
\end{tabular}

A dependência do SUS foi relatada por $58,5 \%$ dos entrevistados. Dado semelhante foi descrito por Scochi et al. [12], em que o SUS tinha em torno de 110 milhóes de usuários, o que correspondia a $60,0 \%$ dos brasileiros [12]. No estudo realizado com motoristas na BR-381 [5], foi observado que 65,7\% dos caminhoneiros só procuravam o SUS quando estavam doentes ou sentindo algum desconforto, e que $38,2 \%$ dos pesquisados nunca fizeram um check-up médico, apresentando como motivos a falta de tempo e dificuldade de se locomover com o caminhão dentro das cidades.

Dos caminhoneiros que náo buscavam o serviço de saúde do SUS, 48 (19,4\%) relataram não ter tempo para ir à UBS, $37(14,9 \%)$ por não terem tido nenhuma doença, $36(14,5 \%)$ preferiam utilizar o plano de saúde particular, $8(3,2 \%)$ afirmaram que o serviço era muito demorado, e $4(1,6 \%)$ foram mal atendidos, o que os levou a náo mais optar pelos serviços do SUS. Segundo relatos de alguns caminhoneiros, "a busca pelo atendimento ocorre nos casos quando o processo da doença já está instalado, pois a falta de tempo entre uma viagem e outra náo permite que eles procurem assistência à saúde".

A busca dos serviços de saúde do SUS como forma de prevençáo foi relatada por $57(23,1 \%)$ dos participantes, $24(9,6 \%)$ náo procuravam as UBS 
em nenhuma circunstância, $2(0,8 \%)$ apenas para controle da pressão arterial, $01(0,4 \%)$ procurava para receber preservativo e outro $(0,4 \%)$ só procurava a UBS em caso de acidente. Segundo alguns relatos, "a náo-procura pelo serviço de saúde do SUS está relacionada à preocupação em não atrasar a viagem, bem como pelo fato dos caminhóes de carga pesada serem proibidos de circular dentro das cidades, dificultando assim o acesso ao Posto de Saúde".

Os caminhoneiros apresentam-se como uma clientela diferenciada para o SUS por não conseguirem aderir às normas e rotinas das UBS, uma vez que o seu trabalho exige grande tempo fora de casa. Somando-se a isto, estes trabalhadores são submetidos a horários irregulares para dormir, alimentar-se e descansar, surgindo para os profissionais de saúde o desafio de planejar estratégias e açóes criativas que visem contemplar essa populaçáo, promovendo sua saúde e prevenindo doenças $[1,5]$.

Ao serem interrogados sobre a presença de algum problema de saúde, $203(81,9 \%)$ negaram qualquer patologia, e dos $45(18,1 \%)$ que relataram possuir algum problema de saúde, $16(6,5 \%)$ mencionaram a hipertensão arterial, $7(2,8 \%)$ o diabetes mellitus, 3 (1,2\%) disseram ter ácido úrico elevado, $3(1,2 \%)$ com bronquite e distúrbio da tireóide, 2 $(0,8 \%)$ com cardiopatias, $2(0,8 \%)$ com o colesterol elevado, $2(0,8 \%)$ com gastrite, $2(0,8 \%)$ com lombalgia, $1(0,4 \%)$ com alergia, $1(0,4 \%)$ com hipoglicemia, $1(0,4 \%)$ com problema psicológico, 1 $(0,4 \%)$ com toxoplasmose, $1(0,4 \%)$ com triglicerídeos elevado e $1(0,4 \%)$ com problemas de vesícula.

Em decorrência dos problemas de saúde relatados, foi questionada qual a necessidade de assistência que eles teriam em função da sua doença. A maioria $(84,6 \%)$ disse não ter nenhuma necessidade de assistência, pois não tinham doenças; já 26 (10,4\%) necessitavam de medicação para controle ou cura da patologia, $8(3,2 \%)$ para o controle da pressão arterial, $6(2,4 \%)$ de exames, 5 (2,0\%) de controle da glicemia, 3 (1,2\%) de acompanhamento da doença e $2(0,8 \%)$ de consulta médica.

Os dados reforçam que os caminhoneiros ainda se orientavam pelo modelo curativo, focado na doença, na investigação, diagnóstico e cura das patologias, e não na prevenção e promoção da sua saúde. De acordo com dados do Ministério da Saúde [13], o modelo atual de promoção à saúde visa a reorganização da prática assistencial em novas bases e critérios, em substituição ao modelo tradicional de assistência (curativo) - orientado somente para a cura de doenças e realizado principalmente nos hospitais - para um modelo que prioriza açóes de promoção a saúde e prevenção.

Ao serem questionados pelo motivo de adesão ao plano de saúde particular, $46,5 \%$ relataram que a empresa na qual eram funcionários ofereciam o benefício; $40,7 \%$ adquiriram o plano para sua segurança e a de seus familiares; $3,8 \%$ relataram que a espera pelo SUS era demorada e o plano oferecia a maior comodidade por náo enfrentar filas; 2,9\% que $o$ atendimento era melhor; $1,9 \%$ afirmaram ter convênio com o seu sindicato e $0,4 \%$ relataram que foi devido ao plano ter maior opção de médicos e dentistas. Segundo Scochi et al. [12], cerca de 21,0\% dos brasileiros usam planos de saúde particulares.

Quando questionados sobre a forma de busca de tratamento para algum problema de saúde, 123 $(49,5 \%)$ disseram ir ao hospital, $73(29,4 \%)$ procuravam o Posto de Saúde, enquanto $27,0 \%$ disseram procurar uma farmácia, conforme demonstrado na Figura 1.

Figura 1 - Distribuição das formas de acesso aos serviços de saúde. Timóteo/MG, 2008

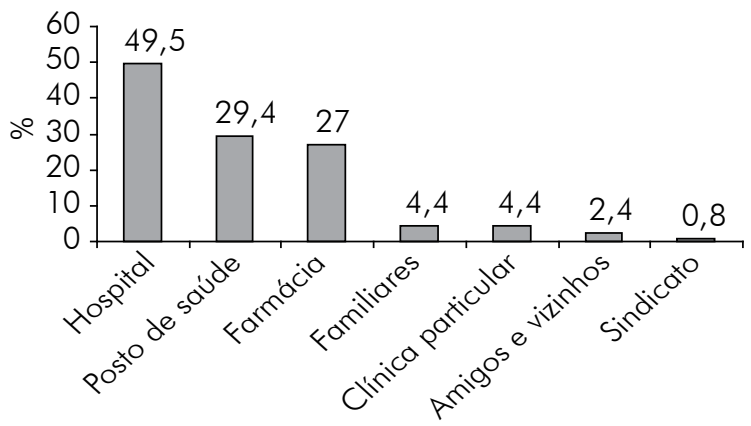

Quando questionados se estavam fazendo algum tratamento, $220(88,7 \%)$ disseram náo estar e $28(11,3 \%)$ afirmaram estar fazendo. Dos que afirmaram, $12(4,8 \%)$ disseram fazer tratamento da hipertensão arterial, $6(21,4 \%)$ do diabetes mellitus, $3(1,2 \%)$ do ácido úrico elevado, $2(0,8)$ odontológico, $1(0,4 \%)$ de bronquite, $1(0,4 \%)$ de cardiopatia, $1(0,4 \%)$ fazia dieta, $1(0,4 \%)$ de distúrbio da tireoide, $1(0,4 \%)$ de gastrite, $1(0,4 \%)$ de tratamento psicológico e $1(0,4 \%)$ de rinite e toxoplasmose. A hipertensáo arterial foi relatada um problema de saúde para 16 caminhoneiros, sendo que apenas 12 faziam tratamento. A hipertensão arterial é considerada um dos principais fatores de risco para a paralisia dos rins, lesóes das artérias e doenças do coração quando náo tratada [14]. 
Ao serem perguntados em qual lugar estavam fazendo o tratamento de saúde, os locais citados foram: 11 (39,5\%) em Posto de Saúde, 8 (28,5\%) em consultório particular, $4(14,2 \%)$ em hospital, $3(10,7 \%)$ com amigos e vizinhos, e $2(7,1 \%)$ com farmacêuticos.

Como demonstrado na Figura 2, a presença de Postos de Saúde nas estradas foi a sugestão mais citada por $22,9 \%$ dos entrevistados para melhorar o atendimento à saúde, sendo que $21,3 \%$ não tinham sugestóes para dar aos profissionais de saúde.

Figura 2 - Sugestões dos caminhoneiros para melhorar o atendimento de saúde. Timóteo-MG, 2008.

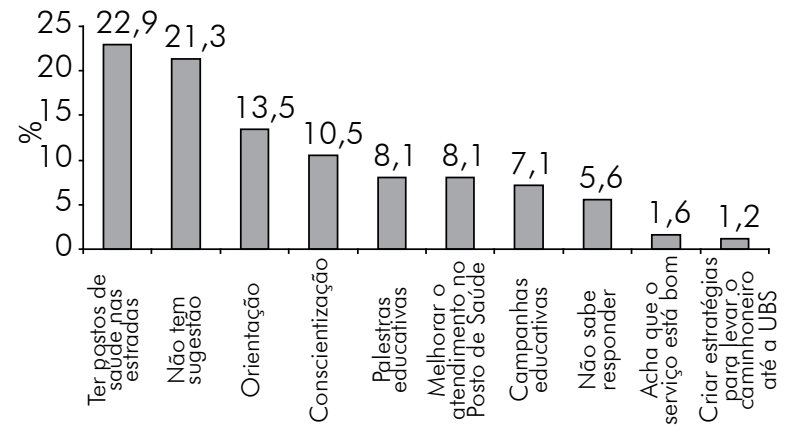

As palestras e orientaçóes são sugestóes importantes na conscientização do caminhoneiro quanto a importância do cuidado com a saúde, uma vez que eles têm excessiva carga horária de trabalho em sua rotina diária, o que muitas vezes se torna um empecilho para que eles busquem informaçóes a respeito de sua saúde. Visando uma melhoria na qualidade de vida desses profissionais, é importante que estas sugestóes sejam transformadas em açôes, de preferência no ambiente de trabalho dos caminhoneiros.

\section{Conclusão}

Embora os caminhoneiros conheçam alguns serviços oferecidos pela Atenção Básica à Saúde, foi relatada dificuldade de acesso a estes serviços devido à exaustiva rotina de trabalho à qual eles são submetidos. É importante repensar na estrutura do funcionamento das Unidades Básica de Saúde subsidiando propostas que visem atender melhor esta clientela, pois o desenvolvimento de medidas que promovam o bem-estar físico, psíquico e social desses profissionais trará benefícios tanto para esta categoria quanto para a toda a sociedade.

\section{Referências}

1. Guedes HM, Brum KA, Costa PA, Almeida MEF. Fatores de risco para o desenvolvimento de hipertensão arterial entre motoristas caminhoneiros. Cogitare Enferm 2010;15(4):652-8.

2. Codarin MAF, Moulatlet EM, Nehme P, Ulhôa M, Moreno CRC. Associaçáo entre prática de atividade física, escolaridade e perfil alimentar de motoristas de caminhão. Saude Soc 2010;19(2):418-28.

3. Nascimento EC, Silva JP, Nascimento E. Uso de álcool e anfetaminas entre caminhoneiros de estrada. Rev Saúde Publica 2007;41(2):290-3.

4. Villarinho L, Bezerra I, Lacerda R, Latorre MRDO, Paiva V, Stall R et al. Caminhoneiros de rota curta e sua vulnerabilidade ao HIV, Santos, SP. Rev Saúde Publica 2002;36(4):61-7.

5. Guedes HM, Paes MSL, Botelho SSP. Uso do "rebite" entre caminhoneiros que trafegam na BR 381 do interior de Minas Gerais: papel do enfermeiro na educaçáo para a saúde. Enfermagem Brasil 2008;7(3):134-40.

6. Cavagioni LC, Pierin AMG, Batista KM, Bianchi ERF, Costa ALS. Agravos à saúde, hipertensão arterial e predisposição ao estresse em motoristas de caminhão. Rev Esc Enferm 2009;43(2):1267-71.

7. Ministério da Saúde. Política Nacional de Atenção Básica. Brasília: MS; 2006.

8. Ministério da Saúde. Conselho Nacional de Saúde. Diretrizes e normas regulamentadoras de pesquisa envolvendo seres humanos. Resolução n. 196, de 10 de outubro de 1996. Brasília: MS; 1996.

9. Penteado RZ, Gonçalves CGO, Costa DD, Marques JM. Trabalho e saúde em motoristas de caminhão no interior de São Paulo. Saúde Soc 2008;17(4):35-45.

10. Souza JC, Paiva T, Reimão R. Qualidade de vida de caminhoneiros. J Bras Psiquiatr 2006;55(3):184-9.

11. Nascimento E. Desenvolvimento de pesquisa-açáo com motoristas de caminhão de estrada: trabalhando na problematização das questóes voltadas a sexualidade: DST/ AIDS e remédios [Tese]. Ribeirão Preto: Universidade de São Paulo; 2003.

12. Scochi MJ, Mathias TAF, Souza RKT, Gazola S, Tavares CRG. Conhecimento e utilizaçáo de serviços de atençáo básica em saúde por famílias em município da região Sul do Brasil. Rev Eletr Enferm 2008;10(2):347-57.

13. Ministério da Saúde. Programa de Saúde da Família. Brasília: MS; 2001.

14. Ministério da Saúde (BR). Pratique saúde contra a hipertensão arterial. Brasília: MS; 2007. 